International Journal of Pure and Applied Mathematics

Volume 100 No. 4 2015, 443-447

ISSN: 1311-8080 (printed version); ISSN: 1314-3395 (on-line version)

url: http://www.ijpam.eu

doi: http://dx.doi.org/10.12732/ijpam.v100i4.1

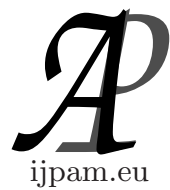

\title{
PROJECTIVE CURVES SUCH THAT A GENERAL POINT OF THE AMBIENT PROJECTIVE IS CONTAINED IN \\ A UNIQUE OSCULATING HYPERPLANE OF THE CURVE
}

\author{
E. Ballico \\ Department of Mathematics \\ University of Trento \\ 38123 Povo (Trento) - Via Sommarive, 14, ITALY
}

\begin{abstract}
We study the non-degenerate integral curves $X \subset \mathbb{P}^{n}$ such that a general point of $\mathbb{P}^{n}$ is contained in a unique osculating hyperplane of $X$ (they are a generalization of the strange curves to the case $n>2$ ).
\end{abstract}

AMS Subject Classification: 14N05, 1499

Key Words: osculating variety, projective curve, strange curve

\section{Introduction}

Let $X \subset \mathbb{P}^{n}, n \geq 2$, be an integral and non-degenerate curve defined over an algebraically closed field $K$. We ask when the natural map from the variety of $(n-1)$-osculating spaces of $X$ to $\mathbb{P}^{n}$ has separable degree 1, i.e. for a general $O \in \mathbb{P}^{n}$ there is a unique osculating hyperplane of $X$ containing $O$. We call A such a property. Set $p:=\operatorname{char}(K)$. It is easy to check that $X$ does not exists if $p=0$ or if $p>\operatorname{deg}(X)$ (Proposition 4 and Remark 1). In positive characteristic we need to choose a definition of osculating variety and osculating space. We use [4], but essentially only use the existence of a non-empty open subset $U \subset X_{\text {reg }}$ and an integer $x \geq n$ such that for each $P \in U$ there is a unique hyperplane $O(X, P, n-1) \subset \mathbb{P}^{n}$ with order of contact $x$ with $U$ at $P$,

Received: October 4, 2014

(c) 2015 Academic Publications, Ltd. url: www.acadpubl.eu 
while all other hyperplanes have order of contact $<x$ with $X$ at $P$. This shows an ambiguity of the definition of $\boldsymbol{\$}$ : unique hyperplane or unique $P \in U$ such that $O \in O(X, P, n-1)$ ? We take the second one as the definition of $\mathbf{p}$, but usually it is easy to modify the proofs to adapt to the other interpretation (we call $\varnothing$ the alternative interpretation). We prove the following results.

Proposition 1. $X$ has property $\odot$ if and only if there is a codimension 2 linear subspace $M \subset \mathbb{P}^{n}$ such that $O(X, P, n-1) \supset M$ for a general $P \in U$. $X$ has $\boldsymbol{Q}$ if and only if it has $\varnothing$ and for a general $P \in U$ the hyperplane $O(X, P, n-1)$ is not an osculating hyperplane of $X$ at another point of $U$.

Proposition 2. Assume $n=2$.

(a) $X$ has property $\odot$ if and only it is a strange curve.

(b) $X$ has property if and only if it is a strange curve and the linear projection from its strange point has separable degree 1.

In particular by a theorem of Lluis ([5]) the case $p=2$ and $X$ a smooth conic is the only case with $n=2, \varnothing$ and $X$ smooth (it also has $\mathbf{\$}$ ).

We recall that there is a construction of all strange plane curves ([3] for $n=2$, [1] when $n>2$ ); in the case $n=2$ it involves the multiplicity $\mu \geq 0$ of $X$ at the strange point, $o$, the separable degree $s$ of the the rational map $\tau$ induced on the normalization of $X$ from the linear projection from $o$ and the inseparable degree $p^{e}$ of $\tau$ (we have $\operatorname{deg}(X)=\mu+s p^{e}$ ). We do not know a way to construct all curve with $\varnothing$ or $\boldsymbol{\phi}$, but we have a way to construct two classes of such curves (see Examples 1 and 2).

\section{The Proofs}

Proposition 3. If $X$ has $\mathbf{n}$, then it is rational, i.e. its normalization has genus 0 .

Proof. Let $f: C \rightarrow X$ be the normalization map. Write $\mathbb{P}^{n}=\mathbb{P}\left(V^{\vee}\right)$ with $V$ an $(n+1)$-dimensional vector space. Let $x$ be the degree of the intersection at a general $P \in X$ of the osculating hyperplane $O(X, P, n-1)$ and $\wp:=P(X, x-$ $\left.1, f^{*}\left(\mathcal{O}_{X}(1)\right)\right)$ the bundle of principal parts of order $x-1$ of the line bundle $f^{*}\left(\mathcal{O}_{X}(1)\right)$. The composition $C \rightarrow X \hookrightarrow \mathbb{P}^{n}$ induces a map $W \otimes \mathcal{O}_{C} \rightarrow \wp$ whose image is a rank $n$ vector bundle $E$ on $C$ and a surjective map $u^{\prime}: \mathbb{P}(E) \rightarrow \mathbb{P}^{n}$ with separable degree one. The projection $\mathbb{P}(E)$ shows that $C$ has genus 0 .

Proof of Proposition 1: First assume that $M$ exists. For any $O \in \mathbb{P}^{n}$ there is a unique hyperplane containing both $M$ and $O$. Since $X$ is non-degenerate, 
we get that $\mathbb{P}^{n}$ is the image of the $(n-1)$-osculating variety of $X$. Hence $X$ has $\varnothing$. Now assume that $X$ has $\varnothing$. Fix a general $O \in \mathbb{P}^{n}$ and take any $P \in U$ with $O \in O(X, P, n-1)$. Let $S \subset X$ be the set of all $Q \in U$ with $O(X, Q, n-1)=O(X, P, n-1) . S$ is a finite set. Fix any $e \in U \backslash S$ and set $M:=O(X, P, n-1) \cap O(X, e, n-1)$. Since $e \notin S$, we have $O(X, e, n-1) \neq$ $O(X, P, n-1)$ and $M$ is a hyperplane of $O(X, P, n-1)$ not containing $O$. Since $X$ has $\varnothing$, then $O \notin O(X, Q, n-1)$ for any $Q \in U \in S$. Since $O$ is general, we see that the union of all $O(X, P, n-1) \cap O(X, Q, n-1), Q \in U \backslash S$ in not Zariski dense in $O(X, P, n-1)$. Therefore $X$ has $\varnothing$ with $M$ as associated codimension two linear subspace. The statement concerning follows from the one concerning $\varnothing$.

Proof of Proposition 2: Part (a) is the case $n=2$ of Proposition 1. Part (b) follows from part (a).

Proposition 4. In characteristic zero there is no curve with $\odot$.

Proof. If $n=2$, just use Proposition 2 and that in characteristic zero no curve, except lines, have a strange point. Now assume $n>2$ and take $M$ as in Proposition 1. We have $x=n-1$ by [4, Theorem 15]. Fix a general $Q \in M$ and call $\ell: \mathbb{P}^{n} \backslash\{Q\} \rightarrow \mathbb{P}^{n-1}$ and set $Y:=\ell(X)$. $Y$ is a non-degenerate curve whose general osculating hyperplane has order of contact $n+1$ with the curve at the contact point, contradicting [4, Theorem 15].

Remark 1. Assume $p>0$. The proof of Proposition 4 and [4, Theorem 15] gives $\operatorname{deg}(X) \geq p$ for each curve $X$ with $\varnothing$.

Let $X \subset \mathbb{P}^{n}$ an integral non-degenerate curve. Fix an open subset $U \subseteq X_{\text {reg }}$ on which the osculating sequence in the sense of [4] is the general one for $X$. Fox each $P \in U$ and each integer $t \in\{1, \ldots, n-1\}$ let $O(X, P, t)$ be the $t$-dimensional linear osculating subspace to $X$ at $P$.

Example 1. We point out how to use [3] and [1] to construct all $X$ with $\odot$ (or $\mathbf{\$})$ for which there are $n-1$ linearly independent points $P_{1}, \ldots, P_{t} \in \mathbb{P}^{n}$ such that for each $t=1, \ldots, n-1$ a general $t$-osculating space of $X$ contains the linear span of $\left\{P_{1}, \ldots, P_{t}\right\}$; call o this property. Note that the construction will not depend, up to a projective equivalence, from the choice of the ordered $(n-1)$-ple of linearly independent points. If $n=2$, then we use Proposition 2 and the construction of strange plane curves done in [3]. Now assume $n>2$ and that the construction is done in $\mathbb{P}^{n-1}$. Let $\ell: \mathbb{P}^{n} \backslash\left\{P_{1}\right\} \rightarrow \mathbb{P}^{n-1}$ be the linear projection from $P_{n}$. Set $O_{i}:=\ell\left(P_{1}\right), 2 \leq i \leq n-1$. The point $O_{2}, \ldots, O_{n-1}$ are linearly independent. Take $Y \subset \mathbb{P}^{n-1}$ with \& with respect to the points 
$O_{2}, \ldots, O_{n-1}$. Fix integer $m \geq 0, s \geq 1$ and $e>0$. The construction in [1] gives all strange curve $X \subset \mathbb{P}^{n}$ with $P_{1}$ as their strange point, with $Y$ as their image by the linear projection $\ell$ and such that $\ell \mid\left(X \backslash\left\{P_{1}\right\}\right.$ has separable degree $s$ and inseparable degree $p^{e}$. Among these curves the ones with are the one for which at each step the separable degree, $s$, is 1 (you need to start a strange plane curve with separable degree 1). The curve discovered by J. Rathmann ([6, Example 1.2]) has (see [2, Example 1] for the explicit equations of $Y$ ).

Example 2. If $n>2$ there are non-strange curves with $\mathbf{n}$. Here there is a way to construct them. Fix a codimension two linear subspace $M \subset \mathbb{P}^{n}$. Choose a system of homogeneous coordinates $x_{0}, \ldots, x_{n}$ such that $M=\left\{x_{0}=x_{1}=0\right\}$. Fix integers $e_{i}>0,1 \leq i \leq n-1$, general $c_{i, j} \in K, 1 \leq i \leq n-1,2 \leq j \leq n$, and general $u_{i}\left(x_{0}, x_{1}\right) \in K\left[x_{0}, x_{1}\right], 1 \leq i \leq n-1$, homogeneous of degree $p^{e_{i}}$. Set $f_{i}\left(x_{0}, \ldots, x_{n}\right):=u_{i}\left(x_{0}, x_{1}\right)+\sum_{j=2}^{n} c_{i, j} x_{j}^{p^{e_{i}}}, X:=\left\{f_{1}=\cdots=f_{n-1}\right.$ and $X_{i}:=\left\{f_{i}\right\}$. For general $f_{1}, \ldots, f_{n-1}$ the scheme $X$ has dimension one and hence it is a locally complete intersection and $\operatorname{deg}(X)=p^{e_{1}+\cdots+e_{n-1}}$. For general $f_{1}, \ldots, f_{n-1}$ we have $X \cap M=\emptyset$ (e.g. if $e_{i}=e$ for all $i$ it is sufficient that the $(n-1) \times(n-1)$ matrix with $c_{i, j}^{1 / e}$ as entries is invertible). The linear projection $\ell: \mathbb{P}^{n} \backslash M \rightarrow \mathbb{P}^{1}$ from $M$ induces a generically bijective $X_{\text {red }} \rightarrow \mathbb{P}^{1}$. Therefore to check that $X$ is an integral curve it is sufficient to check that it is smooth at a general $P \in X$. Fix $P \in X_{\text {reg }}$ and let $H$ be the hyperplane spanned by $P$ and $M$. We have $(H \cap X)_{\text {red }}=\{P\}$, because $M \cap X=\emptyset$ and $\ell \mid X$ has separable degree one. Hence $H$ is the osculating hyperplane to $X$ at $P$.

\section{Acknowledgements}

The author was partially supported by MIUR and GNSAGA of INdAM (Italy).

\section{References}

[1] E. Ballico, On strange projective curves, Rev. Roum. Math. Pures Appl. 37 (1992), 741-745.

[2] E. Ballico, An upper bound for the X-ranks of points of $\mathbb{P}^{n}$ in positive characteristic, Albanian J. Math. 5 (2011), no. 1, 3-10.

[3] V. Bayer and A. Hefez, Strange plane curves, Comm. Algebra 19 (1991), no. $11,3041-3059$. 
[4] D. Laksov, Wronskians and Plücker formulas for linear systems on curves, Ann. Sci. École Norm. Sup. (4) 17 (1984), no. 1, 45-66.

[5] E. Lluis, Variedades algebraicas con ciertas condiciones en sus tangents, Bol. Soc. Mat. Mexicana 7 (1962), 47-56.

[6] J. Rathmann, The uniform position principle for curves in characteristic p, Math. Ann. 276 (1987), no. 4, 565-579. 
